\title{
ANNALES
}

UNIVERSITATIS MARIAE CURIE-SKŁODOWSKA

L UBLIN - POLONIA

VOL. LXV, NO. 2, 2011

SECTIO A

\section{The Schwarz-Pick theorem and its applications}

\author{
This paper is dedicated to the memory of Professor Jan Krzyż
}

\begin{abstract}
Various derivative estimates for functions of exponential type in a half-plane are proved in this paper. The reader will also find a related result about functions analytic in a quadrant. In addition, the paper contains a result about functions analytic in a strip. Our main tool in this study is the Schwarz-Pick theorem from the geometric theory of functions. We also use the Phragmén-Lindelöf principle, which is of course standard in such situations.
\end{abstract}

\section{Introduction and statement of results.}

1.1. An inequality for rational functions. By a result of Bernstein $[2$, p. 339], if $f$ is a polynomial of degree at most $n$ such that $|f(z)| \leq 1$ for $|z|=1$, then

$$
\left|f^{\prime}(z)\right| \leq n|z|^{n-1} \quad(|z|>1)
$$

where $\left|f^{\prime}(z)\right|=n|z|^{n-1}$ at any point $z$ with $|z|>1$ only if $f(z) \equiv \mathrm{e}^{\mathrm{i} \gamma} z^{n}$ for some real $\gamma$. Recently [6, Theorem 1$]$, we have obtained the following analogous result for rational functions which have all their poles inside the unit disk.

2000 Mathematics Subject Classification. 30A10, 30C10, 30C80, 30D15, 41A17.

Key words and phrases. Bernstein's inequality, functions of exponential type in a half-plane, rational functions, Schwarz-Pick theorem. 
Theorem A. Let $f$ be a function analytic (holomorphic) in $\{z \in \mathbb{C}:|z|>$ $\left.\rho_{0}\right\}$ for some $\rho_{0}$ in $(0,1)$, and let $|f(z)| \leq 1$ for $|z|=1$. Suppose, in addition, that, for some $n \in\{ \pm 1, \pm 2, \ldots\}$, the function $f(z) / z^{n}$ tends to a finite limit $L$ as $|z| \rightarrow \infty$ and let

$$
R_{n}:=\sqrt{1+\frac{1}{n^{2}}}+\frac{1}{|n|} .
$$

Then

$$
\left|f^{\prime}(z)\right| \leq|n| R^{n-1} \quad\left(|z|=R \geq R_{n}\right),
$$

and

$$
\left|f^{\prime}(z)\right| \leq \frac{R^{n}}{R^{2}-1}+\frac{1}{4} n^{2} R^{n-2}\left(R^{2}-1\right) \quad\left(1<|z|=R<R_{n}\right) .
$$

The bound in (2) is attained for any $z$ with $|z| \geq R_{n}$ only if $f(z) \equiv \mathrm{e}^{\mathrm{i} \gamma} z^{n}$ for some $\gamma \in \mathbb{R}$. Inequality (3) is also sharp and in fact for each $R \in\left(1, R_{n}\right)$.

One may look at Theorem A as an extension of the above-mentioned result of Bernstein. Of course, (2) is somewhat more restrictive on $|z|$ than (1) but the class of functions to which (2) applies is much wider.

1.2. The role of the Schwarz-Pick theorem. The proof of Theorem A was based on a classical result of G. Pick, also known as the invariant form of Schwarz's Lemma (see $[1$, p. 3], $[4$, p. 41] or $[5, \S 6.2]$ ). It says that if $\phi$ is holomorphic with $|\phi(z)| \leq 1$ for $|z|<1$, then

$$
\left(1-|z|^{2}\right)\left|\phi^{\prime}(z)\right|+|\phi(z)|^{2} \leq 1 \quad(|z|<1) .
$$

It is easily checked that in (4), the equality holds at any given point $\zeta$ of the open unit disk for

$$
\phi(z):=\frac{(z-\zeta)+a(\bar{\zeta} z-1)}{\bar{a}(z-\zeta)+(\bar{\zeta} z-1)} \quad(|a|<1)
$$

and also for any constant of modulus 1 .

It was the late Professor Jan Krzyż who first pointed out to one of us (see the footnote in $[8$, p. 317]), the relevance of (4) to a problem about the coefficients of polynomials. The possibility of applying (4) to obtain inequalities for rational functions does not seem to have been considered before. Novel or not, we find the approach as being a natural one, since the estimates it enabled us to obtain are sharp.

Once we were convinced about the relevance of Pick's result to inequalities for rational functions, we spent some time looking for its known extensions and analogues. We did not come across any that we could use but we ourselves figured out (see [6, p. 74]) the following related proposition involving the first and the second order derivatives. This result may have been known to specialists. We state it here as a lemma since we shall need it later in the paper. 
Lemma 1. Let $\phi$ be holomorphic in the open unit disk and $|\phi(z)| \leq 1$ therein. Then

(5) $\frac{1}{2 !}\left|\left(1-|z|^{2}\right)^{2} \phi^{\prime \prime}(z)-2\left(1-|z|^{2}\right) \bar{z} \phi^{\prime}(z)\right|+|\phi(z)|^{2} \leq 1 \quad(|z|<1)$.

In (5), equality holds at any given point $\zeta$ of the open unit disk for

$$
\phi(z):=\frac{a(1-\bar{\zeta} z)^{2}+(z-\zeta)^{2}}{(1-\bar{\zeta} z)^{2}+\bar{a}(z-\zeta)^{2}} \quad(0<|a|<1)
$$

and also for any constant of modulus 1 .

Lately, we have used (4) to prove the following result [7, Theorem 1].

Proposition 1. Let $g$ be holomorphic in the open upper half-plane and $|g(z)| \leq 1$ therein. Then

$$
2 y\left|g^{\prime}(z)\right|+|g(z)|^{2} \leq 1 \quad(y:=\Im z>0) .
$$

At any given point $z_{0}=x_{0}+\mathrm{i} y_{0}$ with $\Im z_{0}=y_{0}>0$, inequality (6) becomes an equality for

$$
g(z):=\frac{z-z_{0}+a\left(z-\bar{z}_{0}\right)}{\bar{a}\left(z-z_{0}\right)+z-\bar{z}_{0}} \quad(0<|a|<1)
$$

and also for any constant of modulus 1 .

We see inequality (6) more as an analogue of (4) than a consequence of it.

In [7], inequality (6) was applied to obtain an extension of Theorem A to functions of exponential type in a half-plane, stated below as Theorem B. In order to help the reader understand its relationship to Theorem A, we find it desirable to mention certain facts about functions of exponential type. This will also provide a perspective for the new results presented here. However, we need to start with the Phragmén-Lindelöf principle for functions analytic in an angle.

1.3. Functions analytic in an angle and the maximum principle. Let $f$ be holomorphic in the angle $\mathcal{A}\left(\theta_{1}, \theta_{2}\right):=\left\{z=r \mathrm{e}^{\mathrm{i} \theta}: r>0, \theta_{1}<\right.$ $\left.\theta<\theta_{2}\right\}$, where $\theta_{2}-\theta_{1}<2 \pi$. Such a function may not be bounded inside $\mathcal{A}$ if it is bounded on the boundary. Take for example $f(z):=\mathrm{e}^{z^{4}}$. It is an entire function and so holomorphic in the angle $\mathcal{A}(-\pi / 4, \pi / 4)$. Now, note that $|f(z)|=1$ at every point of the boundary but $f(x)=\mathrm{e}^{x^{4}} \rightarrow \infty$ as $x \rightarrow \infty$. This is because the function $f(z):=\mathrm{e}^{z^{4}}$ grows too rapidly inside the angle. Actually, there is a generalization of the maximum principle due to Phragmén and Lindelöf which says that a holomorphic function is bounded inside the angle if it is bounded on the boundary and its growth is not too rapid. It plays an important role in the study of functions holomorphic in an angle and may be stated as follows. For its proof we refer the reader to [9, Theorem 1.6.14]. 
Lemma 2. Let $g$ be analytic in the angle

$$
\mathcal{A}\left(\theta_{0}-\alpha, \theta_{0}+\alpha\right):=\left\{z=r \mathrm{e}^{\mathrm{i} \theta}: r>0,\left|\theta-\theta_{0}\right|<\alpha\right\}, 0<\alpha<\pi,
$$

and continuous on $\overline{\mathcal{A}}\left(\theta_{0}-\alpha, \theta_{0}+\alpha\right)$. Suppose that $|g(z)| \leq M$ on the boundary of the angle, and that, for some $\mu<\pi /(2 \alpha)$ and a sequence $r_{1}, \ldots, r_{n}, \ldots$ tending to infinity, the estimate $g\left(r_{n} \mathrm{e}^{\mathrm{i} \theta}\right)=O\left(e^{r_{n}^{\mu}}\right)$ holds uniformly with respect to $\theta$. Then $|g(z)| \leq M$ throughout $\overline{\mathcal{A}}\left(\theta_{0}-\alpha, \theta_{0}+\alpha\right)$.

1.4. Functions of exponential type. A function $f$, holomorphic in an unbounded region $D$, like a half-plane or more generally an angle, is said to be of exponential type $\tau$ in $D$ if for every $\varepsilon>0$, there exists a constant $K$ depending on $\varepsilon$, but not on $z$, such that

$$
|f(z)|<K \mathrm{e}^{(\tau+\varepsilon)|z|} \quad(z \in D) .
$$

In the case where $D=\mathbb{C}$, a function $f$ satisfying (7) is called an entire function of exponential type $\tau$.

An entire function of exponential type $\tau$ is clearly of exponential type $\tau$ in every angle $\left\{z=r \mathrm{e}^{\mathrm{i} \theta}:\left|\theta-\theta_{0}\right|<\alpha\right\}, 0<\alpha<\pi$.

For an entire function $f$ let $M(r):=\max _{|z|=r}|f(z)|, r>0$. Then $f$ is said to be of order $\rho$ if

$$
\limsup _{r \rightarrow \infty} \frac{\log \log M(r)}{\log r}=\rho .
$$

A constant has order 0 , by convention. The type of an entire function $f$ of positive finite order $\rho$ is defined to be $\lim \sup _{r \rightarrow \infty} r^{-\rho} \log M(r)$. Any entire function of order less than 1 is of exponential type $\tau$ for every $\tau \geq 0$ and so is any entire function of order 1 type at most $\tau$.

To characterize the dependence of the growth of a function $f$ of exponential type $\tau$ in an angle $\left\{z=r \mathrm{e}^{\mathrm{i} \theta}:\left|\theta-\theta_{0}\right|<\alpha\right\}, 0<\alpha<\pi$ on the direction in which $z$ tends to infinity, Phragmén and Lindelöf introduced the function

$$
h_{f}(\theta):=\limsup _{r \rightarrow \infty} \frac{\log \left|f\left(r \mathrm{e}^{\mathrm{i} \theta}\right)\right|}{r} \quad\left(\theta_{0}-\alpha<\theta<\theta_{0}+\alpha\right),
$$

called the indicator function of $f$. It is known that unless $h_{f}(\theta) \equiv-\infty$, $h_{f}(\theta)$ is continuous in $\theta_{0}-\alpha<\theta<\theta_{0}+\alpha$ and that if $\theta_{0}-\alpha<\theta<\theta+\pi<$ $\theta_{0}+\alpha$, then

$$
h_{f}(\theta)+h_{f}(\theta+\pi) \geq 0 .
$$

If $f$ is an entire function of order 1 type $\tau$ then for all $\theta, h_{f}(\theta) \leq \tau$ and so, by $(9), h_{f}(\theta) \geq-\tau$. For these and many other properties of the indicator function, see [3, Chapter 5].

The following lemma [3, Theorem 6.2.4] serves as a basic tool in the study of functions of exponential type. It is not a direct consequence of Lemma 2, but can be deduced from it, as we shall show. 
Lemma 3. Let $f$ be a function of exponential type in the open upper half-plane such that $h_{f}(\pi / 2) \leq c$. Furthermore, let $f$ be continuous in the closed upper half-plane and suppose that $|f(x)| \leq M$ on the real axis. Then

$$
|f(x+\mathrm{i} y)|<M \mathrm{e}^{c y} \quad(-\infty<x<\infty, y>0)
$$

unless $f(z) \equiv M \mathrm{e}^{\mathrm{i} \gamma} \mathrm{e}^{-\mathrm{i} c z}$ for some real $\gamma$.

Proof. Since $f$ is of exponential type, there exist positive constants $A$ and $B$ such that $|f(z)| \leq A \mathrm{e}^{B|z|}$ for all $z$ in the open upper half-plane. Let $g(z):=\mathrm{e}^{\mathrm{i} C z} f(z)$, where $C>c$. Then $|g(x)|=|f(x)| \leq M$ on the real axis. Besides,

$$
|g(\mathrm{i} y)|=\mathrm{e}^{-C y}|f(\mathrm{i} y)| \rightarrow 0 \text { as } y \rightarrow \infty .
$$

Hence, there exists a number $M_{1}$ such that $|g(\mathrm{i} y)| \leq M_{1}$ for all $y \geq 0$ and besides $\left|f\left(\mathrm{i} y_{1}\right)\right|=M_{1}$ for some $y_{1}>0$. Since $|g(z)| \leq A \mathrm{e}^{(B+|C|)|z|}$ for $\Im z>0$, we may apply Lemma 2 to $g$, taking $\theta_{0}=\pi / 4$ and $\alpha=\pi / 4$, to conclude that $|g(z)| \leq \max \left\{M, M_{1}\right\}$ for all $z$ in the first quadrant. When Lemma 2 is applied to $g$ taking $\theta_{0}=3 \pi / 4$ and $\alpha=\pi / 4$, it shows that $|g(z)| \leq \max \left\{M, M_{1}\right\}$ for all $z$ in the second quadrant. Thus, $|g(z)| \leq$ $\max \left\{M, M_{1}\right\}$ for all $z$ in the upper half-plane. However, if $M_{1}$ was larger than $M$, then the maximum of $|g(z)|$ in the closed upper half-plane would be attained at $z=\mathrm{i} y_{1}$, which is possible only if $g$ is a constant of modulus $M_{1}$. This is obviously not the case since $|g(z)|=|f(z)| \leq M$ on the real axis. Thus $|g(z)| \leq M$ for all $z$ in the upper half-plane, which implies that $|f(z)| \leq M \mathrm{e}^{C y}$ for $y:=\Im z>0$. In particular, for any given point $z_{0}=x_{0}+\mathrm{i} y_{0}$ of the open upper half-plane, $\left|f\left(z_{0}\right)\right| \leq M \mathrm{e}^{C y_{0}}$ for any $C>c$. Hence $\left|f\left(z_{0}\right)\right| \leq M \mathrm{e}^{c y_{0}}$ and so $|f(z)| \leq M \mathrm{e}^{c y}$ for all $z$ in the upper halfplane. Equality at any point $z_{0}=x_{0}+\mathrm{i} y_{0}$ of the open upper half-plane would mean that the maximum modulus of the function $\mathrm{e}^{\mathrm{i} c z} f(z)$, which is holomorphic in the open upper half-plane, is attained at an interior point and so the function would be a constant of modulus $M$. It follows that $|f(z)|<M \mathrm{e}^{c y}$ for all $y>0$ unless $f(z) \equiv M \mathrm{e}^{\mathrm{i} \gamma} \mathrm{e}^{-\mathrm{i} c z}$ for some real $\gamma$.

We are now ready to state the analogue (extension) of Theorem A we had alluded to, towards the end of $\S 1.2$. Note that if $f$ satisfies the conditions of Theorem A, then the function $f\left(\mathrm{e}^{-\mathrm{i} z}\right)$ satisfies the conditions of Theorem $B$ with $c=n$.

Theorem B. Let $f$ be a function of exponential type in the open upper half-plane such that

$$
h_{f}\left(\frac{\pi}{2}\right):=\limsup _{y \rightarrow \infty} \frac{\log |f(\mathrm{i} y)|}{|y|} \leq c .
$$

Suppose, in addition, that $f$ is continuous in the closed upper half-plane and that $|f(x)| \leq M$ for all real $x$. If $c \neq 0$, then, for any $w \in \mathbb{C}$, other than 
0, we have

(11) $\mid f^{\prime}(z)+(1+w)$ ic $f(z) \mid \leq \begin{cases}M|w||c| \mathrm{e}^{c y} & \text { if } y:=\Im z \geq 1 /(|w||c|), \\ M \frac{1+|w|^{2} c^{2} y^{2}}{2 y} \mathrm{e}^{c y} & \text { if } 0<y<1 /(|w||c|) .\end{cases}$

If $c=0$, then

$$
\left|f^{\prime}(z)\right| \leq \frac{1}{2 y} M \quad(y>0) .
$$

The first inequality in (11), which holds for $y \geq 1 /(|w||c|)$, becomes an equality for $f(z):=M \mathrm{e}^{\mathrm{i} \gamma} \mathrm{e}^{-\mathrm{i} c z}, \gamma \in \mathbb{R}$. Its proof shows that for any other function satisfying the conditions of Theorem B, it (the first inequality in (11)) is strict for any $z$ with $\Im z \geq 1 /(|w||c|)$.

Let $c \neq 0, w \neq 0$ and let $\zeta=\xi+\mathrm{i} \eta$ be any point of the open upper half-plane such that $-1<w c \eta<1$. Then, with

$$
b:=\eta \frac{1+w c \eta}{1-w c \eta}>0
$$

the function

$$
f(z):=M \mathrm{e}^{\mathrm{i} \gamma} \mathrm{e}^{-\mathrm{i} c(z-\xi)} \frac{(z-\xi)-\mathrm{i} b}{(z-\xi)+\mathrm{i} b} \quad(\gamma \in \mathbb{R})
$$

satisfies the conditions of Theorem B and by a straightforward calculation, we find that

$$
\begin{aligned}
f^{\prime}(\zeta)+(1+w) \mathrm{i} c f(\zeta) & =-M \mathrm{ie}^{\mathrm{i} \gamma} \mathrm{e}^{c \eta}\left\{\frac{2 b}{(\eta+b)^{2}}-w c \frac{\eta-b}{\eta+b}\right\} \\
& =-M \mathrm{i}^{\mathrm{i} \gamma} \mathrm{e}^{c \eta}\left(\frac{1+w^{2} c^{2} \eta^{2}}{2 \eta}\right),
\end{aligned}
$$

which shows that the second inequality in (11) is sharp at least for real $w$.

In order to see that (12) is also sharp, we may take any point $\zeta=\xi+i \eta$ of the open upper half-plane and consider the function

$$
f(z):=M \mathrm{e}^{\mathrm{i} \gamma} \frac{z-\zeta}{z-\bar{\zeta}} .
$$

It satisfies the conditions of Theorem $\mathrm{B}$ with $c=0$. Besides, a simple calculation shows that for this function $\left|f^{\prime}(\zeta)\right|=M /(2 \eta)$.

Here we shall prove some further results about functions of exponential type in a half-plane, involving higher order derivatives. We shall also consider functions holomorphic in an angle of opening $2 \alpha$ for any $\alpha \in(0, \pi)$ and functions holomorphic in a strip. 
1.5. Statement of results. Let $f$ be of exponential type in the open upper half-plane $H$. Is the derivative $f^{\prime}$ also of exponential type in $H$ ? "Not necessarily so" is the answer to this question. To see this, let

$$
\varphi_{n}(z):=\frac{z-\left(n+\mathrm{i}^{-\mathrm{e}^{n}}\right)}{z-\left(n-\mathrm{i}^{-\mathrm{e}^{n}}\right)} \quad(n=1,2, \ldots) .
$$

Then $\left|\varphi_{n}(z)\right| \leq 1$ in the closed upper half-plane $\bar{H}$ for all $n$. Now let

$$
f(z):=\frac{6}{\pi^{2}} \mathrm{e}^{-\mathrm{i} c z} \sum_{n=1}^{\infty} \frac{1}{n^{2}} \varphi_{n}(z) .
$$

It is clear that $|f(z)| \leq \mathrm{e}^{c \Im z}$ for $\Im z \geq 0$ and that

$$
f^{\prime}(z)=\frac{6}{\pi^{2}} \mathrm{e}^{-\mathrm{i} c z}\left\{-\mathrm{i} c \sum_{n=1}^{\infty} \frac{1}{n^{2}} \frac{z-\left(n+\mathrm{ie}^{\left.-\mathrm{e}^{n}\right)}\right.}{z-\left(n-\mathrm{ie}^{\left.-\mathrm{e}^{n}\right)}\right.}+\sum_{n=1}^{\infty} \frac{1}{n^{2}} \frac{2 \mathrm{ie}^{-\mathrm{e}^{n}}}{\left(z-n+\mathrm{ie}^{-\mathrm{e}^{n}}\right)^{2}}\right\} .
$$

Clearly, for $m=1,2, \ldots$, we have

$$
\begin{aligned}
& \left|\sum_{n=1}^{\infty} \frac{1}{n^{2}} \frac{2 \mathrm{ie}^{-\mathrm{e}^{n}}}{\left(z-n+\mathrm{i}^{-\mathrm{e}^{n}}\right)^{2}}\right|_{z=m+\mathrm{ie}^{-\mathrm{e}^{m}}} \\
& \quad=\left|-\mathrm{i} \frac{\mathrm{e}^{\mathrm{e}^{m}}}{2 m^{2}}+\sum_{\substack{n=1, n \neq m}}^{\infty} \frac{1}{n^{2}} \frac{2 \mathrm{ie}^{-\mathrm{e}^{n}}}{\left(m-n+\mathrm{i}^{-\mathrm{e}^{m}}+\mathrm{ie}^{\left.-\mathrm{e}^{n}\right)^{2}}\right.}\right| \geq \frac{1}{2 m^{2}} \mathrm{e}^{\mathrm{e}^{m}}-\frac{\pi^{2}}{3}
\end{aligned}
$$

and

Hence

$$
\left|-\mathrm{i} c \sum_{n=1}^{\infty} \frac{1}{n^{2}} \frac{z-\left(n+\mathrm{i}^{-\mathrm{e}^{n}}\right)}{z-\left(n-\mathrm{i}^{\left.-\mathrm{e}^{n}\right)}\right.}\right|_{z=m+\mathrm{ie}^{-\mathrm{e}^{m}}} \leq|c| \frac{\pi^{2}}{6} .
$$

$$
\left|f^{\prime}\left(m+\mathrm{i}^{-\mathrm{e}^{m}}\right)\right| \geq \frac{6}{\pi^{2}} \mathrm{e}^{c \mathrm{e}^{-\mathrm{e}^{m}}}\left\{\frac{1}{2 m^{2}} \mathrm{e}^{\mathrm{e}^{m}}-\frac{\pi^{2}}{6}(|c|+2)\right\},
$$

which shows that $f^{\prime}$ is far from being of exponential type in the open upper half-plane although $|f(z)| \leq \mathrm{e}^{c \Im z}$ for any $z$ in the closed upper half-plane. This example underscores the interest of the following result implied by Theorem B.

Lemma 4. Let $f$ be analytic in the open upper half-plane $H$ and suppose that $|f(z)| \leq \mathrm{e}^{c \Im z}$ for all $z \in \bar{H}$. Then $f^{\prime}$ is of exponential type $c$ in the half-plane $\mathfrak{H}_{y_{0}}:=\left\{z: \Im z>y_{0}\right\}$ for any $y_{0}>0$.

In fact, it follows from (11) that for any $z$ in $\mathfrak{H}_{y_{0}},\left|f^{\prime}(z)\right| \leq|c| \mathrm{e}^{c y}$ if $y_{0} \geq 1 /|c|$, whereas $\left|f^{\prime}(z)\right| \leq y_{0}^{-1} \mathrm{e}^{c y}$ if $y_{0}<1 /|c|$.

In Theorem 1, we present an upper bound for $\left|f^{(k)}(z)\right|$ at any given point $z$ of the open upper half-plane. 
Theorem 1. Let $f$ be a function of exponential type in the open upper half-plane such that $h_{f}(\pi / 2) \leq c \neq 0$. Suppose, in addition, that $f$ is continuous in the closed upper half-plane and that $|f(x)| \leq M$ for all real $x$. Then, for $k=1,2,3, \ldots$, we have

$$
\left|f^{(k)}(z)\right| \leq \begin{cases}M|c|^{k} \mathrm{e}^{c y} & \text { if } y:=\Im z \geq k /|c|, \\ M\left(\frac{k^{2}+c^{2} y^{2}}{2 k y}\right)^{k} \mathrm{e}^{c y} & \text { if } 0<y<k /|c| .\end{cases}
$$

How good is this bound? For $k=1$, it is the best point-wise bound. The example $f(z):=M \mathrm{e}^{-\mathrm{i} c z}$ shows that for any $k \geq 2$ we can say the same for points $z$ with $\Im z \geq k /|c|$.

If $k \geq 2$ and $0<y \leq k /|c|$ then, by (13), we have

$$
\left|f^{(k)}(x+\mathrm{i} y)\right| \leq M\left(\frac{k^{2}+c^{2} y^{2}}{2 k y}\right)^{k} \mathrm{e}^{c y} \sim M\left(\frac{k}{2}\right)^{k} \frac{1}{y^{k}} \mathrm{e}^{c y} \text { as } y \downarrow 0 .
$$

Here, we only claim that the dependence of the bound on $y$ is the right one. To see this, let $\xi+\mathrm{i} \eta$ be any point with $\eta>0$ and consider the function

$$
f(z):=M \mathrm{e}^{-\mathrm{i} c(z-\xi)}\left(\frac{z-\xi-\mathrm{i} \eta}{z-\xi+\mathrm{i} \eta}\right)^{k}=M(z-\xi-\mathrm{i} \eta)^{k} \cdot \frac{\mathrm{e}^{-\mathrm{i} c(z-\xi)}}{(z-\xi+\mathrm{i} \eta)^{k}} .
$$

Using Leibnitz' rule to calculate $f^{(k)}(z)$ and then putting $z=\xi+\mathrm{i} \eta$, we find that

$$
\begin{aligned}
\left|f^{(k)}(\xi+\mathrm{i} \eta)\right| & =M \frac{k !}{2^{k}} \frac{1}{\eta^{k}} \mathrm{e}^{c \eta} \\
& =M\left(\frac{k}{2}\right)^{k} \frac{1}{\eta^{k}} \mathrm{e}^{c \eta} \mathrm{e}^{-k}(2 \pi k)^{1 / 2} \mathrm{e}^{\delta /(12 k)}, \quad 0<\delta<1,
\end{aligned}
$$

by Stirling's formula.

In the next theorem we consider two special differential operators.

Theorem 2. Let $f$ be a function of exponential type in the open upper half-plane such that $h_{f}(\pi / 2) \leq c \neq 0$. Suppose, in addition, that $f$ is continuous in the closed upper half-plane and that $|f(x)| \leq M$ for all real $x$. Finally, for $y:=\Im z>0$, let

$$
\Lambda_{j}[f(z)]:= \begin{cases}y^{2} f^{\prime \prime}(z)-\mathrm{i} y f^{\prime}(z)+\mathrm{i} c y\left\{2 y f^{\prime}(z)-\mathrm{i} f(z)\right\} & \text { if } j=1, \\ y^{2} f^{\prime \prime}(z)-\mathrm{i} y f^{\prime}(z)+2 \mathrm{i} c y^{2}\left\{f^{\prime}(z)+\mathrm{i}(c / 2) f(z)\right\} & \text { if } j=2 .\end{cases}
$$

Then

$$
\left|\Lambda_{1}[f(z)]\right| \leq \begin{cases}M c^{2} y^{2} \mathrm{e}^{c y} & \text { if } y \geq 1 /|c| \\ M \frac{1+c^{4} y^{4}}{2} \mathrm{e}^{c y} & \text { if } 0<y<1 /|c|\end{cases}
$$


and

$$
\left|\Lambda_{2}[f(z)]\right| \leq \begin{cases}M|c| y \mathrm{e}^{c y} & \text { if } y \geq 1 /|c|, \\ M \frac{1+c^{2} y^{2}}{2} \mathrm{e}^{c y} & \text { if } 0<y<1 /|c| .\end{cases}
$$

The example $f(z):=M \mathrm{e}^{-\mathrm{i} c z}$ shows that the upper bound for $\left|\Lambda_{1}[f(z)]\right|$, given in (14), is sharp for $y:=\Im z \geq 1 /|c|$. The same can be said about the upper bound for $\left|\Lambda_{2}[f(z)]\right|$ given in (15).

If a function $f$ satisfies the conditions of Theorem 2 and $0<y \leq 1 /|c|$, then by inequalities (14) and (15), we have

$$
\left|\Lambda_{1}[f(x+\mathrm{i} y)]\right| \leq \frac{M}{2} \mathrm{e}^{c y}+\frac{M}{2} c^{4} y^{4} \mathrm{e}^{c y}=\frac{M}{2} \mathrm{e}^{c y}+O\left(y^{4}\right) \text { as } y \downarrow 0
$$

and

$$
\left|\Lambda_{2}[f(x+\mathrm{i} y)]\right| \leq \frac{M}{2} \mathrm{e}^{c y}+\frac{M}{2} c^{2} y^{2} \mathrm{e}^{c y}=\frac{M}{2} \mathrm{e}^{c y}+O\left(y^{2}\right) \text { as } y \downarrow 0,
$$

respectively. On the other hand,

$$
f(z):=M \mathrm{e}^{-\mathrm{i} c(z-\xi)}\left(\frac{z-\xi-\mathrm{i} \eta}{z-\xi+\mathrm{i} \eta}\right)^{2}, \eta>0
$$

satisfies the conditions of Theorem 2 and for this function

$$
\left|\Lambda_{1}[f(\xi+\mathrm{i} \eta)]\right|=\left|\Lambda_{2}[f(\xi+\mathrm{i} \eta)]\right|=\frac{M}{2} \mathrm{e}^{c \eta} .
$$

2. An auxiliary result. For the proof of Theorem 2 we need the following result which is to be compared with (6).

Lemma 5. Let $g$ be holomorphic in the open upper half-plane and $|g(z)| \leq 1$ therein. Then

$$
2\left|y^{2} g^{\prime \prime}(z)-\mathrm{i} y g^{\prime}(z)\right|+|g(z)|^{2} \leq 1 \quad(y:=\Im z>0) .
$$

Example 1. For any $z_{0}$ with $y_{0}:=\Im z_{0}>0$ and any $a$ such that $0<|a|<1$, let

$$
B(z):=\left(\frac{z-z_{0}}{z-\bar{z}_{0}}\right)^{2} \text { and } g(z):=\frac{a+B(z)}{1+\bar{a} B(z)} .
$$

Then

$$
B^{\prime}(z)=2 \frac{z-z_{0}}{z-\bar{z}_{0}} \frac{2 \mathrm{i} y_{0}}{\left(z-\bar{z}_{0}\right)^{2}} \text { and } g^{\prime}(z)=\frac{\left(1-|a|^{2}\right) B^{\prime}(z)}{(1+\bar{a} B(z))^{2}}
$$

so that

$$
g^{\prime}\left(z_{0}\right)=0 .
$$

Furthermore,

$$
B^{\prime \prime}(z)=2\left(\frac{2 \mathrm{i} y_{0}}{\left(z-\bar{z}_{0}\right)^{2}}\right)^{2}+2 \frac{z-z_{0}}{z-\bar{z}_{0}} \frac{-4 \mathrm{i} y_{0}}{\left(z-\bar{z}_{0}\right)^{3}}
$$


and

$$
g^{\prime \prime}(z)=\left(1-|a|^{2}\right)\left\{\frac{B^{\prime \prime}(z)}{(1+\bar{a} B(z))^{2}}-2 \bar{a} \frac{\left(B^{\prime}(z)\right)^{2}}{(1+\bar{a} B(z))^{3}}\right\}
$$

so that

$$
g^{\prime \prime}\left(z_{0}\right)=-\left(1-|a|^{2}\right) \frac{1}{2 y_{0}^{2}} .
$$

Hence

$$
2\left|y_{0}^{2} g^{\prime \prime}\left(z_{0}\right)-\mathrm{i} y_{0} g^{\prime}\left(z_{0}\right)\right|+\left|g\left(z_{0}\right)\right|^{2}=\left|-\left(1-|a|^{2}\right)\right|+|a|^{2}=1,
$$

which shows that inequality (16) becomes an equality for the function

$$
g(z):=\frac{a+\left(\frac{z-z_{0}}{z-\bar{z}_{0}}\right)^{2}}{1+\bar{a}\left(\frac{z-z_{0}}{z-\bar{z}_{0}}\right)^{2}}=\frac{\left(z-z_{0}\right)^{2}+a\left(z-\bar{z}_{0}\right)^{2}}{\bar{a}\left(z-z_{0}\right)^{2}+\left(z-\bar{z}_{0}\right)^{2}}
$$

when $z=z_{0}$. Of course, the equality holds also for any constant of modulus 1 .

Proof of Lemma 5. The function

$$
\phi(\zeta):=g\left(\frac{1+\zeta}{1-\zeta} \mathrm{i}\right)
$$

is holomorphic in the open unit disk $|\zeta|<1$ and $|\phi(\zeta)| \leq 1$ therein. In order to prove (16), we shall apply Lemma 1 to $\phi$ for which we need to calculate $\phi^{\prime}(\zeta)$ and $\phi^{\prime \prime}(\zeta)$. Note that

$$
\phi^{\prime}(\zeta)=\frac{2 \mathrm{i}}{(1-\zeta)^{2}} g^{\prime}\left(\frac{1+\zeta}{1-\zeta} \mathrm{i}\right)
$$

and

$$
\phi^{\prime \prime}(\zeta)=\left(\frac{2 \mathrm{i}}{(1-\zeta)^{2}}\right)^{2} g^{\prime \prime}\left(\frac{1+\zeta}{1-\zeta} \mathrm{i}\right)+\frac{4 \mathrm{i}}{(1-\zeta)^{3}} g^{\prime}\left(\frac{1+\zeta}{1-\zeta} \mathrm{i}\right) .
$$

Hence, by (5), we obtain

$$
\begin{aligned}
& \frac{1}{2} \mid\left(1-|\zeta|^{2}\right)^{2}\left\{\frac{-4}{(1-\zeta)^{4}} g^{\prime \prime}\left(\frac{1+\zeta}{1-\zeta} \mathrm{i}\right)+\frac{4 \mathrm{i}}{(1-\zeta)^{3}} g^{\prime}\left(\frac{1+\zeta}{1-\zeta} \mathrm{i}\right)\right\} \\
& \quad-\left.2\left(1-|\zeta|^{2}\right) \bar{\zeta} \frac{2 \mathrm{i}}{(1-\zeta)^{2}} g^{\prime}\left(\frac{1+\zeta}{1-\zeta} \mathrm{i}\right)|+| g\left(\frac{1+\zeta}{1-\zeta} \mathrm{i}\right)\right|^{2} \leq 1 \quad(|\zeta|<1) .
\end{aligned}
$$

This leads us to the inequality

$$
\begin{gathered}
2 \frac{1}{|1-\zeta|^{4}}\left|\left(1-|\zeta|^{2}\right)^{2} g^{\prime \prime}\left(\frac{1+\zeta}{1-\zeta} \mathrm{i}\right)-\mathrm{i}\left(1-|\zeta|^{2}\right)\left(1-\zeta-\bar{\zeta}+|\zeta|^{2}\right) g^{\prime}\left(\frac{1+\zeta}{1-\zeta} \mathrm{i}\right)\right| \\
+\left|g\left(\frac{1+\zeta}{1-\zeta} \mathrm{i}\right)\right|^{2} \leq 1 \quad(|\zeta|<1) .
\end{gathered}
$$


Now set

$$
z:=\frac{1+\zeta}{1-\zeta} \mathrm{i} \quad \text { so that } \quad \zeta=\frac{z-\mathrm{i}}{z+\mathrm{i}}
$$

Hence

$$
1-\zeta=\frac{2 \mathrm{i}}{z+\mathrm{i}}
$$

and

$$
\begin{aligned}
1-\zeta-\bar{\zeta}+|\zeta|^{2} & =1-\zeta-\bar{\zeta}(1-\zeta)=(1-\zeta)(1-\bar{\zeta}) \\
& =|1-\zeta|^{2}=\frac{4}{|z+\mathrm{i}|^{2}} .
\end{aligned}
$$

The preceding inequality can therefore be written as

$$
\frac{1}{8}\left|\left(|z+\mathrm{i}|^{2}-|z-\mathrm{i}|^{2}\right)^{2} g^{\prime \prime}(z)-4 \mathrm{i}\left(|z+\mathrm{i}|^{2}-|z-\mathrm{i}|^{2}\right) g^{\prime}(z)\right|+|g(z)|^{2} \leq 1 \text {. }
$$

Now, in order to obtain (16), it suffices to note that

$$
\begin{aligned}
|z+\mathrm{i}|^{2}-|z-\mathrm{i}|^{2} & =(z+\mathrm{i})(\bar{z}-\mathrm{i})-(z-\mathrm{i})(\bar{z}+\mathrm{i})=2 \mathrm{i}(\bar{z}-z) \\
& =4 \Im z=4 y .
\end{aligned}
$$

\section{Proofs of Theorems 1 and 2.}

Proof of Theorem 1. Taking $w=-1$ in (11), we see that (13) holds for $k=1$. For a proof by induction, let us assume that (13) holds for $k=j$, i.e.

$$
\left|f^{(j)}(z)\right| \leq \begin{cases}M|c|^{j} \mathrm{e}^{c y} & \text { if } y:=\Im z \geq j /|c| \\ M\left(\frac{j^{2}+c^{2} y^{2}}{2 j y}\right)^{j} \mathrm{e}^{c y} & \text { if } 0<y<j /|c| .\end{cases}
$$

It suffices to show that if (17) holds, then we must necessarily have

$$
\left|f^{(j+1)}(z)\right| \leq \begin{cases}M|c|^{j+1} \mathrm{e}^{c y} & \text { if } y:=\Im z \geq(j+1) /|c| \\ M\left(\frac{(j+1)^{2}+c^{2} y^{2}}{2(j+1) y}\right)^{j+1} \mathrm{e}^{c y} & \text { if } 0<y<(j+1) /|c| .\end{cases}
$$

If $0<\eta<(j+1) /|c|$, then $0<\eta j /(j+1)<j /|c|$ and so by the second inequality in (17), we have

$$
\begin{aligned}
\left|f^{(j)}\left(x+\mathrm{i} \frac{j}{j+1} \eta\right)\right| & \leq M\left(\frac{j^{2}+c^{2} \eta^{2} j^{2} /(j+1)^{2}}{2 \eta j^{2} /(j+1)}\right)^{j} \exp \left(c \frac{j}{j+1} \eta\right) \\
& =M\left(\frac{1+c^{2} \eta^{2} /(j+1)^{2}}{2 \eta /(j+1)}\right)^{j} \exp \left(c \frac{j}{j+1} \eta\right) .
\end{aligned}
$$

Since $\eta-\eta j /(j+1)=\eta /(j+1)<1 /|c|$, we may apply (11) with $w=-1$, to the function

$$
f^{(j)}\left(z+\mathrm{i} \frac{j}{j+1} \eta\right)
$$


to conclude that

$\left|f^{(j+1)}(x+\mathrm{i} \eta)\right| \leq \sup _{x \in \mathbb{R}}\left|f^{(j)}\left(x+\mathrm{i} \frac{j}{j+1} \eta\right)\right| \frac{1+c^{2} \eta^{2} /(j+1)^{2}}{2 \eta /(j+1)} \exp \left(c \frac{1}{j+1} \eta\right)$.

Combining the two preceding inequalities, we obtain

$$
\left|f^{(j+1)}(x+\mathrm{i} \eta)\right| \leq\left(\frac{(j+1)^{2}+c^{2} \eta^{2}}{2(j+1) \eta}\right)^{j+1} \mathrm{e}^{c \eta} \quad\left(0<\eta<\frac{j+1}{|c|}\right),
$$

which is the same as the second inequality in (18).

It is left to the reader to verify that the first inequality in (17) implies the first inequality in (18).

Proof of Theorem 2. Without loss of generality we may suppose that $M=1$. Suppose, in addition, that $f(z)$ is not of the form $\mathrm{e}^{\mathrm{i} \gamma} \mathrm{e}^{-\mathrm{i} c z}$ for any real $\gamma$. Then, in view of Lemma $3,|f(x+\mathrm{i} y)|<\mathrm{e}^{c y}$ for all $y>0$. Applying (16) to the function $g(z):=\mathrm{e}^{\mathrm{i} c z} f(z)$, we see that

(19) $2\left|y^{2} f^{\prime \prime}(z)+\mathrm{i} y(2 c y-1) f^{\prime}(z)-c y(c y-1) f(z)\right| \mathrm{e}^{-c y}+|f(z)|^{2} \mathrm{e}^{-2 c y} \leq 1$

for $y:=\Im z>0$. Therefore, under the same restriction on $y$, we have

$2\left|y^{2} f^{\prime \prime}(z)+\mathrm{i} y(2 c y-1) f^{\prime}(z)+c y f(z)\right| \leq \mathrm{e}^{c y}-\mathrm{e}^{c y}\left(\mathrm{e}^{-c y}|f(z)|\right)^{2}+2 c^{2} y^{2}|f(z)|$.

Now, note that

$$
\mathrm{e}^{c y}-\mathrm{e}^{-c y}|f(z)|^{2}+2 c^{2} y^{2}|f(z)|<2 c^{2} y^{2} \mathrm{e}^{c y}
$$

if and only if

$$
\mathrm{e}^{-c y}\left(\mathrm{e}^{2 c y}-|f(z)|^{2}\right)<2 c^{2} y^{2}\left(\mathrm{e}^{c y}-|f(z)|\right) \quad(y>0) .
$$

Since $\mathrm{e}^{c y}-|f(z)|>0$ for $y>0$, the preceding inequality holds if and only if

$$
\mathrm{e}^{-c y}\left(\mathrm{e}^{c y}+|f(z)|\right)<2 c^{2} y^{2},
$$

that is, if and only if

$$
|f(z)|<\mathrm{e}^{c y}\left(2 c^{2} y^{2}-1\right) .
$$

Once again, because $|f(z)|<\mathrm{e}^{c y}$ for $y>0$, this latter inequality is certainly satisfied for $y \geq 1 /|c|$. Thus, if $y \geq 1 /|c|$, then

$$
2\left|y^{2} f^{\prime \prime}(z)+\mathrm{i} y(2 c y-1) f^{\prime}(z)+c y f(z)\right|<2 c^{2} y^{2} \mathrm{e}^{c y},
$$

which completes the proof of the first part of (14). We have in fact proved that the inequality is strict unless $f(z)$ is of the form $M \mathrm{e}^{\mathrm{i} \gamma} \mathrm{e}^{-\mathrm{i} c z}$ for some real $\gamma$.

Now let $0<y<1 /|c|$. In order to prove the second part of (14), we set

$$
\Psi_{1}(y ; t):=1+2 c^{2} y^{2} t-t^{2}
$$

and write (19) in the form

$$
2\left|y^{2} f^{\prime \prime}(z)+\mathrm{i} y(2 c y-1) f^{\prime}(z)+c y f(z)\right| \leq \mathrm{e}^{c y} \Psi_{1}\left(y ; \mathrm{e}^{-c y}|f(z)|\right) .
$$


Let us suppose that $f(z) \not \equiv \mathrm{e}^{\mathrm{i} \gamma} \mathrm{e}^{-\mathrm{i} c z}$ for any real $\gamma$. Then $t:=\mathrm{e}^{-c y}|f(z)|<$ 1 for $\Im z=y>0$ and we need to determine how large the continuous function $\Psi_{1}(y ; t)$ can be if $t$ belongs to $[0,1)$. Clearly, $\Psi_{1}(y ; t)$ is maximized for $t=c^{2} y^{2}$ and $\Psi_{1}(y ; t) \leq 1+c^{4} y^{4}$ for $0 \leq t<1$. Hence

$$
\left|\Lambda_{1}[f(x+\mathrm{i} y)]\right| \leq \frac{1+c^{4} y^{4}}{2} \mathrm{e}^{c y} \quad\left(0<y<\frac{1}{|c|}\right) .
$$

In order to prove (15), we define

$$
\Psi_{2}(y ; t):=1+2|c| y t-t^{2},
$$

where $t$ varies in $[0,1]$ and $y$ is a parameter. Clearly, (19) can be written as

$$
2\left|y^{2} f^{\prime \prime}(z)-\mathrm{i} y f^{\prime}(z)+2 \mathrm{i} c y^{2}\left\{f^{\prime}(z)+\mathrm{i} \frac{c}{2} f(z)\right\}\right| \leq \Psi_{2}\left(y ; \mathrm{e}^{-c y}|f(z)|\right)
$$

and therefore, we look for the largest value that $\Psi_{2}(y, t)$ can take if $y$ is a given positive number and $t$ varies in $[0,1]$. If $y \geq 1 /|c|$, then

$$
\frac{\partial}{\partial t} \Psi_{2}(y ; t)>0 \text { for } 0 \leq t<1
$$

and so $\Psi_{2}(y ; t)<\Psi_{2}(y ; 1)$ for any $t$ in $[0,1)$. Since $t=\mathrm{e}^{-c y}|f(z)|<1$ for $y:=\Im z>0$, unless $f(z) \equiv \mathrm{e}^{\mathrm{i} \gamma} \mathrm{e}^{-\mathrm{i} c z}$ for some $\gamma \in \mathbb{R}$, it follows that if $f$ satisfies the conditions of Theorem 2 with $M=1$ and $f(z) \not \equiv \mathrm{e}^{\mathrm{i} \gamma} \mathrm{e}^{-\mathrm{i} c z}$ for any $\gamma \in \mathbb{R}$, then

$$
\left|y^{2} f^{\prime \prime}(z)-\mathrm{i} y f^{\prime}(z)+2 \mathrm{i} c y^{2}\left\{f^{\prime}(z)+\mathrm{i} \frac{c}{2} f(z)\right\}\right|<|c||y| \mathrm{e}^{c y} \quad\left(y \geq \frac{1}{|c|}\right) .
$$

The proof of the second part of (15) is left to the reader.

\section{Two other unbounded regions.}

4.1. Functions analytic in an angle of opening $<\mathbf{2 \pi}$. Let $g$ be a function of exponential type in the open right half-plane such that $h_{g}(0) \leq c$. Suppose, in addition, that $g$ is continuous in the closed right half-plane and that $|g(z)| \leq M$ on the imaginary axis. If $c \neq 0$, then Theorem B may be applied to the function $f(z):=g(-\mathrm{i} z)$ to conclude that

$$
\left|g^{\prime}(z)\right| \leq \begin{cases}M|c| \mathrm{e}^{c x} & \text { if } x:=\Re z \geq 1 /|c|, \\ M \frac{1+c^{2} x^{2}}{2 x} \mathrm{e}^{c x} & \text { if } 0<x<1 /|c| .\end{cases}
$$

Now, let $f(\zeta)$ be a function analytic in $\mathcal{A}(-\alpha, \alpha)$, where the reader may refer back to $\S 1.3$ for the definition of $\mathcal{A}\left(\theta_{1}, \theta_{2}\right)$. Suppose, in addition, that

$$
|f(\zeta)|<A \mathrm{e}^{B|\zeta|^{\pi /(2 \alpha)}}, A>0, B>0 \quad(\zeta \in \mathcal{A}(-\alpha, \alpha))
$$

and that

$$
\limsup _{\xi \rightarrow \infty} \frac{\log |f(\xi)|}{\xi^{\pi /(2 \alpha)}} \leq c
$$


Furthermore, let $f$ be continuous on $\overline{\mathcal{A}}(-\alpha, \alpha)$ and let $\left|f\left(\rho \mathrm{e}^{ \pm \mathrm{i} \alpha}\right)\right| \leq M$.

Taking that branch of $z^{2 \alpha / \pi}$ which associates with the point $z=r \mathrm{e}^{\mathrm{i} \theta}$ of the open right half-plane the point $\zeta=\rho \mathrm{e}^{\mathrm{i} \varphi}$ of $\mathcal{A}(-\alpha, \alpha)$, let $g(z):=$ $f\left(z^{2 \alpha / \pi}\right)$. Then, $g$ is a function of exponential type in the open right half-plane and $h_{g}(0) \leq c$. Besides, $g$ is continuous in the closed right half-plane and $|g(z)| \leq M$ on the imaginary axis. Hence, if $c \neq 0$, then (20) holds for the function $g(z):=f\left(z^{2 \alpha / \pi}\right)$, which means that

$$
\left|\frac{\mathrm{d} \zeta}{\mathrm{d} z}\right|\left|f^{\prime}(\zeta)\right|=\left|g^{\prime}(z)\right| \leq \begin{cases}M|c| \mathrm{e}^{c x} & \text { if } \quad x:=\Re z \geq 1 /|c|, \\ M \frac{1+c^{2} x^{2}}{2 x} \mathrm{e}^{c x} & \text { if } 0<x<1 /|c| .\end{cases}
$$

Thus

$$
\frac{2 \alpha}{\pi}|z|^{\left(\frac{2 \alpha}{\pi}\right)-1}\left|f^{\prime}\left(z^{\frac{2 \alpha}{\pi}}\right)\right| \leq \begin{cases}M|c| \mathrm{e}^{c x} & \text { if } x:=\Re z \geq 1 /|c|, \\ M \frac{1+c^{2} x^{2}}{2 x} \mathrm{e}^{c x} & \text { if } 0<x<1 /|c| .\end{cases}
$$

Since $z^{2 \alpha / \pi}=\rho \mathrm{e}^{\mathrm{i} \varphi}$, as stipulated above, we have

$$
|z|=\rho^{\pi /(2 \alpha)} \text { and } x=\rho^{\frac{\pi}{2 \alpha}} \cos \left(\frac{\pi \varphi}{2 \alpha}\right) .
$$

Hence, the following result holds.

Theorem 3(a). Let $f(\zeta)$ be analytic in $\mathcal{A}(-\alpha, \alpha)$ for some $\alpha<\pi$. Suppose that (21) holds and that (22) is satisfied for some $c \neq 0$. Suppose, in addition, that $f$ is continuous on $\overline{\mathcal{A}}(-\alpha, \alpha)$ and that $\left|f\left(r \mathrm{e}^{ \pm \mathrm{i} \alpha}\right)\right| \leq 1$. Then, at any point $\zeta=\rho \mathrm{e}^{\mathrm{i} \varphi}$ of $\mathcal{A}(-\alpha, \alpha)$, we have

$$
\left|f^{\prime}(\zeta)\right| \leq \begin{cases}\frac{\pi}{2 \alpha}|c| \rho^{\frac{\pi}{2 \alpha}}-1 \exp \left(c \rho^{\frac{\pi}{2 \alpha}} \cos \frac{\pi \varphi}{2 \alpha}\right) & \text { if } \rho^{\frac{\pi}{2 \alpha}} \cos \frac{\pi \varphi}{2 \alpha} \geq \frac{1}{|c|} \\ \frac{\pi}{2 \alpha} \frac{1+c^{2} \rho^{\frac{\pi}{\alpha}} \cos ^{2} \frac{\pi \varphi}{2 \alpha}}{2 \rho \cos \frac{\pi \varphi}{2 \alpha}} \exp \left(c \rho^{\frac{\pi}{2 \alpha}} \cos \frac{\pi \varphi}{2 \alpha}\right) & \text { if } 0<\rho^{\frac{\pi}{2 \alpha}} \cos \frac{\pi \varphi}{2 \alpha}<\frac{1}{|c|} .\end{cases}
$$

Proposition 1 implies that if $g$ is holomorphic in the open right half-plane and $|g(z)| \leq 1$ therein, then

$$
2 x\left|g^{\prime}(z)\right|+|g(z)|^{2} \leq 1 \quad(x:=\Re z>0) .
$$

Hence, if $f(\zeta)$ is holomorphic in $\mathcal{A}(-\alpha, \alpha)$ and $|f(\zeta)| \leq 1$ therein, then applying the preceding inequality to the function $g(z):=f\left(z^{2 \alpha / \pi}\right)$, we obtain the following result, which may be seen as the Schwarz-Pick theorem for an angle.

Proposition 2. Let $f$ be holomorphic in the angle $\mathcal{A}(-\alpha, \alpha)$ and $|f(\zeta)| \leq 1$ therein. Then

$$
\frac{4 \alpha}{\pi} \rho\left(\cos \frac{\pi \varphi}{2 \alpha}\right)\left|f^{\prime}(\zeta)\right|+|f(\zeta)|^{2} \leq 1 \quad\left(\zeta=\rho \mathrm{e}^{\mathrm{i} \varphi} \in \mathcal{A}(-\alpha, \alpha)\right) .
$$


Let $g$ be holomorphic and of exponential type in the open right half-plane such that $h_{g}(0) \leq 0$. Furthermore, let $g$ be continuous in the closed right half-plane and suppose that $|g(z)| \leq 1$ on the imaginary axis. Then, Lemma 3 implies that $|g(z)|<1$ in the open right half-plane unless $g(z) \equiv$ $\mathrm{e}^{\mathrm{i} \gamma}$ for some real $\gamma$. This may be applied to $g(z):=f\left(z^{2 \alpha / \pi}\right)$ to conclude that if $f$ satisfies all the conditions of Theorem 3(a) except that (22) holds with $c=0$, then $|f(\zeta)| \leq 1$ for all $\zeta \in \mathcal{A}(-\alpha, \alpha)$. We thus obtain the following supplement to Theorem 3(a).

Theorem 3(b). Let $f(\zeta)$ be analytic in $\mathcal{A}(-\alpha, \alpha)$ for some $\alpha<\pi$. Suppose that (21) is satisfied and that (22) holds with $c=0$. In addition, let $f$ be continuous on $\overline{\mathcal{A}}(-\alpha, \alpha)$ and suppose that $\left|f\left(r \mathrm{e}^{ \pm \mathrm{i} \alpha}\right)\right| \leq 1$. Then (24) holds.

In view of this result, inequality (24) can be seen as a counterpart of (23). The following result is obtained on combining Proposition 2 with Lemma 2.

Corollary 1. Let $f(\zeta)$ be analytic in the angle $\mathcal{A}(-\alpha, \alpha), 0<\alpha<\pi$ and continuous on $\overline{\mathcal{A}}(-\alpha, \alpha)$. Suppose that $|f(\zeta)| \leq 1$ on the boundary of the angle, and that, for some $\mu<2,|f(\zeta)|<A \mathrm{e}^{B|\zeta|^{\mu}}, A>0, B>0$ for all $\zeta \in \mathcal{A}(-\alpha, \alpha)$. Then (24) holds.

Here, $\mu=2$ is inadmissible as the example $f(\zeta):=\mathrm{e}^{\zeta^{2}}$ shows.

Inequality (24) takes a particularly simple form in the case where $\alpha=$ $\pi / 4$. In that case, the angle $\mathcal{A}(-\alpha, \alpha)$ is a quadrant - a case that has some special significance.

Corollary 2. Let $f$ be holomorphic in the angle $\mathcal{A}(-\pi / 4, \pi / 4)$ and $|f(\zeta)|$ $\leq 1$ therein. Then

$$
\rho(\cos 2 \varphi)\left|f^{\prime}(\zeta)\right|+|f(\zeta)|^{2} \leq 1 \quad\left(\zeta=\rho \mathrm{e}^{\mathrm{i} \varphi} \in \mathcal{A}(-\pi / 4, \pi / 4)\right) .
$$

Remark. Inequality (25) is sharp. Given any point

$$
\zeta_{0}=\rho_{0} \mathrm{e}^{\mathrm{i} \varphi_{0}} \in \mathcal{A}(-\pi / 4, \pi / 4)
$$

and any number $a \in \mathbb{C}$ such that $0 \leq|a|<1$, the function

$$
f(\zeta)=\frac{\zeta^{2}-\zeta_{0}^{2}+a\left(\zeta^{2}+\bar{\zeta}_{0}^{2}\right)}{\bar{a}\left(\zeta^{2}-\zeta_{0}^{2}\right)+\left(\zeta^{2}+\bar{\zeta}_{0}^{2}\right)}
$$

satisfies all the conditions of Corollary 2. Clearly, $f\left(\zeta_{0}\right)=a$. Besides, it is a matter of simple verification that

$$
\left|f^{\prime}\left(\zeta_{0}\right)\right|=\frac{1}{\rho_{0} \cos 2 \varphi_{0}}\left(1-|a|^{2}\right) .
$$


In order to be helpful to the reader we wish to point out that $\zeta_{0}^{2}$ lies in the open right half-plane and so does $\zeta^{2}$. Since $-\bar{\zeta}_{0}^{2}$ is the reflection of $\zeta_{0}^{2}$ in the imaginary axis, the number

$$
\frac{\zeta^{2}-\zeta_{0}^{2}}{\zeta^{2}+\bar{\zeta}_{0}^{2}}
$$

lies in the open unit disk. Now, writing

$$
\frac{\zeta^{2}-\zeta_{0}^{2}+a\left(\zeta^{2}+\bar{\zeta}_{0}^{2}\right)}{\bar{a}\left(\zeta^{2}-\zeta_{0}^{2}\right)+\left(\zeta^{2}+\bar{\zeta}_{0}^{2}\right)}=\frac{\frac{\zeta^{2}-\zeta_{0}^{2}}{\zeta^{2}+\bar{\zeta}_{0}^{2}}+a}{\bar{a} \frac{\zeta^{2}-\zeta_{0}^{2}}{\zeta^{2}+\bar{\zeta}_{0}^{2}}+1},
$$

where $|\bar{a}|<1$, we see that the function $f(\zeta)$ of (26) cannot have any singularities in $\mathcal{A}(-\pi / 4, \pi / 4)$.

4.2. Functions analytic in a strip. Proposition 1 also leads us to the following result which can be seen as the Schwarz-Pick theorem for a strip. Similar results involving higher order derivatives can also be proved, but we shall not do that here.

Proposition 3. Let $f(\zeta)$ be analytic in the vertical strip

$$
\mathcal{S}(-b, b):=\{\zeta=\xi+\mathrm{i} \eta:-b<\xi<b\}
$$

such that $|f(\zeta)| \leq 1$ for all $\zeta \in \mathcal{S}(-b, b)$. Then

$$
\frac{4 b}{\pi} \cos \left(\frac{\pi}{2 b} \xi\right)\left|f^{\prime}(\zeta)\right|+|f(\zeta)|^{2} \leq 1 \quad(\zeta \in \mathcal{S}(-b, b)) .
$$

Given any $a \in \mathbb{C}, 0 \leq|a| \leq 1$ and any point $\zeta_{0}=\xi_{0}+\mathrm{i} \eta_{0} \in \mathcal{S}(-b, b)$, let

$$
f(\zeta):=\frac{\mathrm{e}^{-\mathrm{i} \frac{\pi}{2 b} \zeta}-\mathrm{e}^{-\mathrm{i} \frac{\pi}{2 b} \zeta_{0}}+a\left(\mathrm{e}^{-\mathrm{i} \frac{\pi}{2 b} \zeta}+\mathrm{e}^{\mathrm{i} \frac{\pi}{2 b} \bar{\zeta}_{0}}\right)}{\bar{a}\left(\mathrm{e}^{-\mathrm{i} \frac{\pi}{2 b} \zeta}-\mathrm{e}^{-\mathrm{i} \frac{\pi}{2 b} \zeta_{0}}\right)+\mathrm{e}^{-\mathrm{i} \frac{\pi}{2 b} \zeta}+\mathrm{e}^{\mathrm{i} \frac{\pi}{2 b} \bar{\zeta}_{0}}} .
$$

It is easily checked that $f$ satisfies the conditions of Proposition 3. Besides,

$$
f\left(\zeta_{0}\right)=a \quad \text { and } \quad f^{\prime}\left(\zeta_{0}\right)=-\mathrm{i} \frac{\pi}{4 b}\left(1-|a|^{2}\right) \mathrm{e}^{-\mathrm{i} \frac{\pi}{2 b} \xi_{0}} \sec \left(\frac{\pi}{2 b} \xi_{0}\right)
$$

so that

Hence (27) is sharp.

$$
\frac{4 b}{\pi} \cos \left(\frac{\pi}{2 b} \xi_{0}\right)\left|f^{\prime}\left(\zeta_{0}\right)\right|+\left|f\left(\zeta_{0}\right)\right|^{2}=1 .
$$

Proof. As the point $\zeta=\xi+\mathrm{i} \eta$ describes the straight line

$$
\{\zeta=\xi+\mathrm{i} \eta: \xi \in(-b, b),-\infty<\eta<\infty\}
$$

in the $\zeta$-plane, the point $z=\mathrm{e}^{-\mathrm{i}\left(\frac{\pi}{2 b} \zeta-\frac{\pi}{2}\right)}$ in the $z$-plane describes the ray

$$
\left\{z=r \mathrm{e}^{\mathrm{i} \theta}: \theta=\frac{\pi}{2}\left(1-\frac{\xi}{b}\right), 0<r<\infty\right\},
$$


where $r$ is an increasing function of $\eta$. For $z$ in the open upper half-plane, let $\log z=\ln |z|+\mathrm{i} \arg z$, where $0<\arg z<\pi$. Then, there is a one-to-one correspondence between the point $z$ of the open upper half-plane and the point

$$
\zeta=\mathrm{i} \frac{2 b}{\pi} \log z+b
$$

of $\mathcal{S}(-b, b)$. Since $f$ is analytic in the strip $\mathcal{S}(-b, b)$ and $|f(\zeta)| \leq 1$ therein, the function

$$
g(z):=f\left(\mathrm{i} \frac{2 b}{\pi} \log z+b\right)
$$

is analytic in the open upper half-plane and $|g(z)| \leq 1$ therein. Proposition 1 applies and shows that

(29) $\frac{4 b}{\pi} y \mathrm{e}^{-\frac{\pi}{2 b} \eta}\left|f^{\prime}\left(\mathrm{i} \frac{2 b}{\pi} \log z+b\right)\right|+\left|f\left(\mathrm{i} \frac{2 b}{\pi} \log z+b\right)\right|^{2} \leq 1(\Im z>0)$.

Clearly,

$$
y:=\Im z=\Im\left\{\mathrm{e}^{-\mathrm{i}\left(\frac{\pi}{2 b} \zeta-\frac{\pi}{2}\right)}\right\}=\Re\left\{\mathrm{e}^{-\frac{\mathrm{i} \pi}{2 b}(\xi+\mathrm{i} \eta)}\right\}=\mathrm{e}^{\frac{\pi}{2 b} \eta} \cos \left(\frac{\pi}{2 b} \xi\right) .
$$

Hence, in view of (28), inequality (29) is equivalent to (27).

Corollary 3. As in Proposition 3, let $f$ be analytic in the vertical strip $\mathcal{S}(-b, b)$ wherein $|f(\zeta)| \leq A \mathrm{e}^{\mathrm{e}^{B|\eta|}}$ for some $A>0$ and some $B<\pi /(2 b)$. Also let $f$ be continuous on the closure $|\Re \zeta| \leq b$ of $\mathcal{S}(-b, b)$ and suppose that $|f(\zeta)| \leq 1$ on its boundary $\xi=\Re \zeta= \pm b$. Then (27) holds. Here, $B=\pi /(2 b)$ is inadmissible.

Proof. The result is obvious if $f \equiv 0$ and so let $f$ be not identically zero. In view of Proposition 3, we need to prove that if $f$ is analytic in $\mathcal{S}(-b, b)$, wherein $|f(\zeta)| \leq A \mathrm{e}^{\mathrm{e}^{B|\eta|}}, A>0, B<\pi / 2 b$ and $|f(\xi)| \leq 1$ for $\xi= \pm b$, then $|f(\zeta)| \leq 1$ for $|\xi| \leq b$. For this let us consider the function

$$
F(\zeta):=f(\zeta) \mathrm{e}^{-\delta \mathrm{e}^{-\mathrm{iC} \zeta}}, \delta>0, B<C<\frac{\pi}{2 b}
$$

in the half-strip $S_{+}(-b, b):=\{\zeta=\xi+\mathrm{i} \eta:|\xi|<b, \eta \geq 0\}$. Then

$|F(\zeta)| \leq A \mathrm{e}^{\mathrm{e}^{B \eta}}\left|\mathrm{e}^{-\delta \mathrm{e}^{-\mathrm{i} C(\xi+\mathrm{i} \eta)}}\right|=A \mathrm{e}^{\mathrm{e}^{B \eta}} \mathrm{e}^{-\delta \mathrm{e}^{C \eta} \cos C \xi} \leq A \mathrm{e}^{-\left\{\delta(\cos C b) \mathrm{e}^{C \eta}-\mathrm{e}^{B \eta}\right\}}$

and so, for any $\varepsilon>0$, there exists a number $\eta_{\varepsilon}$ such that $|F(\zeta)|<1$ for all $\zeta \in \mathcal{S}_{+}(-b, b)$ such that $\eta=\Im \zeta>\eta_{\varepsilon}$. Hence, if $|F(\xi)| \leq M$ for $-b \leq \xi \leq b$, then $|F(\zeta)| \leq \max \{1, M\}$ for all $\zeta$ in $\overline{\mathcal{S}}_{+}(-b, b)$. Applying this to the function $\overline{F(\bar{z})}$, we conclude that $|F(\zeta)| \leq \max \{1, M\}$ for all $\zeta$ such that $|\Re \zeta| \leq b$ and $\Im \zeta \leq 0$. Thus $|F(\zeta)| \leq \max \{1, M\}$ for all $\zeta \in \overline{\mathcal{S}}(-b, b)$. However, $M$ cannot be larger than 1 since otherwise the maximum of $|F(\zeta)|$ over $\overline{\mathcal{S}}(-b, b)$ would be attained at an interior point 
and $F$ would be a constant $\not \equiv 0$ and $|f(\mathrm{i} \eta)| \mathrm{e}^{-\mathrm{e}^{B \eta}}$ would tend to $\infty$ with $\eta$, which would be a contradiction. Thus,

$$
|f(\zeta)| \leq\left|\mathrm{e}^{\delta \mathrm{e}^{-\mathrm{i} C \zeta}}\right| \quad(\zeta \in \overline{\mathcal{S}}(-b, b)) .
$$

Since this is true for any $\delta>0$, we must have $|f(\zeta)| \leq 1$ for all $\zeta \in \overline{\mathcal{S}}(-b, b)$.

The conclusion does not hold if $B$ is allowed to be $\pi /(2 b)$. In fact, if

$$
f(\zeta):=\exp \left(\mathrm{e}^{-\mathrm{i} \frac{\pi}{2 b} \zeta}\right)
$$

then $|f( \pm b+\mathrm{i} \eta)|=1$ for $-\infty<\eta<\infty$ whereas $f(\mathrm{i} \eta)=\exp \left(\mathrm{e}^{\frac{\pi}{2 b} \eta}\right) \rightarrow \infty$ as $\eta \rightarrow \infty$.

Remark. Proposition 3 says in particular that if $\phi(z)$ is analytic in the vertical strip $-1<x:=\Re z<1$ and $|\phi(z)| \leq 1$ therein, then

$$
\frac{4}{\pi} \cos \left(\frac{\pi}{2} x\right)\left|\phi^{\prime}(z)\right|+|\phi(z)|^{2} \leq 1 \quad(|z|<1) .
$$

Comparing this with (4), we see that here the condition on $\phi$ is more restrictive, but the conclusion is stronger. To be sure about the conclusion being stronger, we need to check that

$$
\frac{4}{\pi} \cos \left(\frac{\pi}{2} x\right) \geq 1-\left(x^{2}+y^{2}\right)=1-|z|^{2} \quad(|z|<1) .
$$

In fact, we shall show that

$$
x^{2}+\frac{4}{\pi} \cos \frac{\pi}{2} x \geq 1 \quad(-1 \leq x \leq 1) .
$$

It is known that $(\sin \theta) / \theta>2 / \pi$ for $0<\theta<\pi / 2$ and so

$$
x-\sin \frac{\pi}{2} x<0 \text { for } 0<x<1 .
$$

Consequently

$$
\frac{\mathrm{d}}{\mathrm{d} x}\left(x^{2}+\frac{4}{\pi} \cos \frac{\pi}{2} x\right)=2\left(x-\sin \frac{\pi}{2} x\right)<0 \text { for } 0<x<1,
$$

which implies that

$$
x^{2}+\frac{4}{\pi} \cos \frac{\pi}{2} x \geq\left[x^{2}+\frac{4}{\pi} \cos \frac{\pi}{2} x\right]_{x=1}=1 \quad(-1 \leq x \leq 1) .
$$

The following result is a special case of (30).

Corollary 4. Let $\phi(z)$ be analytic in the vertical strip $-1<x:=\Re z<1$ and let $|\phi(z)| \leq 1$ therein. Furthermore, let $\phi(z):=\sum_{k=0}^{\infty} a_{k} z^{k}$ be the Maclaurin expansion of $\phi(z)$ in the open unit disk. Then

$$
\frac{4}{\pi}\left|a_{1}\right|+\left|a_{0}\right|^{2} \leq 1
$$




\section{REFERENCES}

[1] Ahlfors, L. V., Conformal Invariants: Topics in Geometric Function Theory, McGraw-Hill Book Company, New York-Düsseldorf-Johannesburg, 1973.

[2] Bernstein, S. N., Sur la limitation des dérivées des polynomes, C. R. Math. Acad. Sci. Paris 190 (1930), 338-340.

[3] Boas, Jr., R. P., Entire Functions, Academic Press, New York, 1954.

[4] Carathéodory, C., Conformal Representation, Cambridge Tracts in Mathematics and Mathematical Physics, No. 28, Cambridge University Press, Cambridge, 1963.

[5] Krzyż, J. G., Problems in Complex Variable Theory, American Elsevier Publishing Company, Inc., New York, 1971.

[6] Qazi, M. A., Rahman, Q. I., Some estimates for the derivatives of rational functions, Comput. Methods Funct. Theory 10 (2010), 61-79.

[7] Qazi, M. A., Rahman, Q. I., Functions of exponential type in a half-plane, Complex Var. Elliptic Equ. (in print).

[8] Rahman, Q. I., Inequalities concerning polynomials and trigonometric polynomials, J. Math. Anal. Appl. 6 (1963), 303-324.

[9] Rahman, Q. I., Schmeisser, G., Analytic Theory of Polynomials, Clarendon Press, Oxford, 2002.

\author{
M. A. Qazi \\ Department of Mathematics \\ Tuskegee University \\ Tuskegee, AL 36088 \\ U.S.A. \\ e-mail: qazima@aol.com
}

Received June 27, 2011

\section{Q. I. Rahman}

Département de Mathématiques et de Statistique

Université de Montréal

Montréal, Québec H3C 3J7

Canada

e-mail: rahmanqi@dms.umontreal.ca 